\title{
UCRL-TR-235145
}

LAW REN CE LIVERMORE N A TIO N A L LABORATORY
VACET Software Engineering Group's progress reports: May 2007

H. R. Childs

October 1, 2007 
This document was prepared as an account of work sponsored by an agency of the United States government. Neither the United States government nor Lawrence Livermore National Security, LLC, nor any of their employees makes any warranty, expressed or implied, or assumes any legal liability or responsibility for the accuracy, completeness, or usefulness of any information, apparatus, product, or process disclosed, or represents that its use would not infringe privately owned rights. Reference herein to any specific commercial product, process, or service by trade name, trademark, manufacturer, or otherwise does not necessarily constitute or imply its endorsement, recommendation, or favoring by the United States government or Lawrence Livermore National Security, LLC. The views and opinions of authors expressed herein do not necessarily state or reflect those of the United States government or Lawrence Livermore National Security, LLC, and shall not be used for advertising or product endorsement purposes.

This work performed under the auspices of the U.S. Department of Energy by Lawrence Livermore National Laboratory under Contract DE-AC52-07NA27344. 


\title{
VACET Software Engineering Group's Progress Report: May $\underline{2007^{1}}$
}

\author{
Hank Childs, VACET Chief Software Engineer \\ June 4, 2007
}

Each document in this series will summarize a teleconference of the VACET Software Engineering Group (SEG), which in turn summarizes the progress of the previous month. For May 2007, the teleconference took place on June $4^{\text {th }}$.

\begin{tabular}{|l|l|}
\hline \multicolumn{1}{|c|}{ Participant } & \multicolumn{1}{c|}{ Organization } \\
\hline Sean Ahern & ORNL \\
\hline Hank Childs & LLNL \\
\hline Marty Cole & UUtah \\
\hline Jeremy Meredith & ORNL \\
\hline Gunther Weber & LBL \\
\hline
\end{tabular}

\section{Organizational updates:}

There are no formal organizational updates. However, Ken Joy has confirmed that Christoph Garth will serve as a member of the SEG when he starts at UC Davis in July.

\section{Technical updates:}

The teleconference consisted of a series of site reports from each of the four participating sites. Technical discussions about the work being done at each site took place during each site report.

\section{LBL update:}

\footnotetext{
${ }^{1}$ This document is UCRL-TR-229828.
} 
Gunther continued work on the Surface LIC plot. His current plan is to use a resampled grid for the texture, but working on the full resolution of the data when extracting an isosurface.

Gunther also added two of Cristina's existing plugins to the VisIt repository: h5part and nimrod.

\section{LLNL update:}

Hank made good progress on migrating the VisIt project to Subversion. Most team members are now using Subversion to do development and this transition is nearing completion.

Hank did miscellaneous work for various customers:

1) He made progress on the analysis he is doing for Marc Day.

2) He fixed a bug where resampling vectors was producing garbage results. This created problems for Gunther's surface LIC work.

3) Hank interacted with Tech-X and helped them with writing a file format reader.

\section{ORNL update:}

Jeremy spent time improving the build process under Subversion. Previously, under ClearCase, dependencies were resolved using the ClearCase specific tool "clearmake." As we now use gmake, Jeremy improved the dependency tracking.

Also, Jeremy starting building and playing with Chombo and is making early progress on getting Chombo to connect to VisIt from within gdb.

Finally, Jeremy worked on port forwarding issues, since VisIt was unable to connect client-server for customers at ORNL.

UCDavis update: There are currently no members of the SEG from UCDavis.

\section{UUtah update:}


Marty has continued to productize SLIVR. There is now a stand-alone library that passes regression tests. That library is accessible to both SCIRun \& Uuenta. He is getting ready to check Mac \& Windows. We spent some time discussing the plan for merging SLIVR into VisIt. We also discussed volume rendering papers that address distributed memory parallel algorithms in this space.

(Hank's note: the notes from this telecom were not typed up until several months after the telecom. I don't think this document captures everything discussed in the telecon, and more importantly, the work that went on during this period. But this is particularly true for Marty's report, as he was very busy getting SLIVR to work during this period.) 


\title{
VACET Software Engineering Group's Progress Report: July $\underline{2007^{2}}$
}

\author{
Hank Childs, VACET Chief Software Engineer \\ July 30, 2007
}

Each document in this series will summarize a teleconference of the VACET Software Engineering Group (SEG), which in turn summarizes the progress of the previous month. For June and July 2007, the teleconference took place on July $30^{\text {th }}$. (The June teleconference was scheduled during the SciDAC conference and was combined with this one.)

\begin{tabular}{|l|l|}
\hline \multicolumn{1}{|c|}{ Participant } & \multicolumn{1}{c|}{ Organization } \\
\hline Hank Childs & LLNL \\
\hline Marty Cole & UUtah \\
\hline Jeremy Meredith & ORNL \\
\hline Gunther Weber & LBL \\
\hline
\end{tabular}

\section{Organizational updates:}

Christophe Garth has been named the UCD SEG lead. Christophe was unable to call in today because of travel delays.

\section{Technical updates:}

The teleconference consisted of a series of site reports from each of the four participating sites. Technical discussions about the work being done at each site took place during each site report.

\section{LBL update:}

\footnotetext{
${ }^{2}$ This document is UCRL-TR-XXXXX.
} 
Gunther has gotten the SurfaceLIC plugin for VisIt working. He believes that it could be included as an experimental plugin in VisIt. (Hank agrees.) Although AMR data is the target, Gunther took a resampling approach that will allow this plot to work on more data set types than just AMR. Gunther has recently started adding caching capabilities, which will greatly improve performance. The current caching is specific to the SurfaceLIC plot, but more general caching capability will be considered in the future. Gunther and Wes plan to demonstrate this capability to John Bell and then decide on future directions.

Louis Feng is back from Pixar. Gunther suggests that he could work at generating good streamline seedpoints for AMR data. This is needed functionality.

Gunther is interested in running VisIt on the Cray machine "franklin". Sean knows the most about this, so we decide to wait until the next telecon.

\section{LLNL update:}

VisIt has been transitioned to Subversion. All development on the previous repository system, ClearCase, has stopped, and development is only being done on the Subversion server provided by the SciDAC Outreach Center. The release process for VisIt version 1.6.1 was done using Subversion with minimal complications. This work was done primarily by Hank Childs as a joint ASC/SciDAC effort, but Hank was supported by Subversion experts Jeremy Meredith of ORNL and Gunther Weber of LBL.

Hank has continued on his analysis work for Marc Day. Some progress was made over the last two months, but this project was put aside for other deliverables. It is one of Hank's top three priorities for August.

Kathleen Bonnell implemented selection of AMR grids by logical coordinates in VisIt. With this feature, a user can select all of a region within some logical range. For example, a user can select "refinement level 2 , indices $\mathrm{I}=0$ through $\mathrm{I}=250, \mathrm{~J}=15$ through $\mathrm{J}=25$ ", which would return patches at all refinement levels (not just level 2) that fit within the physical space defined by those logical coordinates. This implementation matches that of ChomboVis. 
Brad Whitlock implemented a feature referred to by the Chombo group as "visitrc". ChomboVis has a ".chomborc" file that makes common operations accessible through a single button click. This facility is also customizable. Brad implemented a similar facility in VisIt, vetting his proposed implementation through Terry Ligocki. The implementation leverages VisIt's command line interface and allows for .visitrc files to be generated by one end user and then given to another. This should be helpful for creating a .visitrc that is similar to the .chomborc file and distributing that to existing ChomboVis users to ease their transition.

Hank provided support to Tech-X developers who are developing readers for FACETs. They ran into several development problems, especially dealing with third party libraries and shared libraries, and Hank was able to get them on track.

Hank started integrating the SLIVR library into VisIt. This library was successfully extracted from SCIRun by Marty Cole and Marty has helped Hank through the integration process. This process is still in an early stage.

Hank presented a talk and paper and the SciDAC conference in Boston in June on upcoming problems and solutions for petascale visualization and analysis.

Finally, Hank has made good progress on speeding up VisIt's coloring of patches and levels. VisIt's existing implementation is too general, meaning its performance and memory footprint is lacking. The check-in for the streamlined implementation is expected by August $1^{\text {st }}$, at which time the initial iteration of VisIt changes targeted to roll out to the Chombo group will be done.

\section{ORNL update:}

Jeremy Meredith worked on real time debugging infrastructure for the Chombo project. His work was in two parts. First, he extended VisIt so that it could be driven through the command line interface by a running simulation. Second, he familiarized himself with Chombo and learned how to modify it for doing real time debugging with tools other than ChomboVis. He has gotten ChomboVis to use VisIt for its debugging use cases (bringing up a visual spreadsheet, etc). This code has not been integrated back into the main Chombo repo, however. Further, before doing so, Jeremy would like 
to add support for choosing between VisIt and ChomboVis with an environment variable and also work on configure/autoconf type support. That said, Jeremy feels he is very close to being done with this project. Jeremy will pursue the final changes with Terry Ligocki. He will be in California in late August and may try to roll this feature out then (along with Gunther Weber, as the customer representative for the Chombo group).

Sean Ahern gave a tutorial with Allen Sanderson at the SciDAC conference in Boston.

Finally, Jeremy Meredith has worked on adding support for user selectable options for reading and writing of files in VisIt. Previously, VisIt would automatically make choices about how to interpret the various arrays in a file (e.g. this array is the $\mathrm{X}$-coordinates, this is the ' $\mathrm{Y}$ ', etc.). When possible, this is a good strategy, because it spares users from having to input this knowledge. However, user input is sometimes necessary, and Jeremy added infrastructure to VisIt to obtain this input. While doing this work, Jeremy found and fixed several bugs where VisIt was confusing information about which plugins were available on the client and which were available on the server. In addition to aiding his own work, this bug fix was requested by Tech-X and they were pleased when it was fixed. The final interface for providing options for reading and writing files is still in progress. The current version is functional, but more infrastructure is needed to support anticipated future needs and Jeremy is investigating the scope of this infrastructure.

UCDavis update: Christophe has just joined the SEG, so there is no progress from UCD this month.

\section{UUtah update:}

Marty finalized the SLIVR library and has made it available to Hank. In addition, he has put together example programs so that Hank can see how to use the library. Hank is now the bottleneck going forward.

Marty has also been active in modifying VisTrails to support Dean Williams and the CDAT community. The API for CDAT is very large, so Marty has started with wrapping some simple yet commonly used test scripts. He has some basic functionality working as of the time of the teleconference. His 
plan is to put in place enough functionality that the CDAT community can make a fair assessment of VisTrails. He smartly wants to understand the extent that they are interested in the tool before going further, although Marty in enthused about the possibilities. (Hank spoke with Dean at the SciDAC conference and Dean also expressed a lot of enthusiasm.) Marty also wants to understand the directions they are interested in going and invest in those areas. He hopes that the CDAT community will aid in the wrapping once VisTrails viability is established. Marty is directly interfacing with Dean Williams. 


\title{
VACET Software Engineering Group's Progress Report: $\underline{\text { August 2007 }}$
}

\author{
Hank Childs, VACET Chief Software Engineer \\ September 6, 2007
}

Each document in this series will summarize a teleconference of the VACET Software Engineering Group (SEG), which in turn summarizes the progress of the previous month. For August 2007, the teleconference took place on September $6^{\text {th }}$.

\begin{tabular}{|l|l|}
\hline \multicolumn{1}{|c|}{ Participant } & \multicolumn{1}{c|}{ Organization } \\
\hline Sean Ahern & ORNL \\
\hline Wes Bethel & LBL \\
\hline Hank Childs & LLNL \\
\hline Marty Cole & UUtah \\
\hline Christoph Garth & UCD \\
\hline Jeremy Meredith & ORNL \\
\hline Gunther Weber & LBL \\
\hline Brad Whitlock & LLNL \\
\hline
\end{tabular}

\section{Technical updates:}

The teleconference consisted of a series of site reports from each of the the five sites. Technical discussions about the work being done at each site took place during each site report.

\section{LBL update:}

\footnotetext{
${ }^{3}$ This document is UCRL-TR-XXXXX.
} 
Gunther reported good progress in getting VisIt deployed to APDEC. Previously, we had held back on delivering VisIt, pending the completion of the activities outlined in the PMP. Since those activities (rather the first wave of activities) are now complete, we started the roll out in a serious way. Brian van Straalen, APDEC's point of contact, told Gunther, "we are now your users, you are going to suffer." In an effort to support APDEC, Gunther set up build environments for both the PowerPPC Mac and the IntelMac. He also deployed beta releases of VisIt that contained features important to APDEC that were added after release 1.6.1. These changes included a Boundary operator, as well as material volume fraction support for Chombo files. In addition to leading the roll out for the APDEC team, Gunther supplied a collaborator of theirs, Greg Miller of UC Davis, with the beta release.

Gunther and Hank also collaborated on some changes to enable APDEC to start using VisIt. They include:

1) Making the clip operator work with the plane tool, as per request from Brian van Straalen

2) Pursuing a bug where ghost data was being lost, which turned out to be due to new functionality were compressed files could be read directly.

3) Making the clip operator and slice operator work together using the plane tool, as per request from Brian van Straalen

4) Make the pick functionality automatically bring up a spreadsheet of the region, as per request from Brian van Straalen. This particular effort took several weeks of collective time between Gunther and Hank, both to add infrastructure to VisIt and also to perform GUI work, where picks are highlighted to show exactly what was picked.

5) A boundary operator, which isolates the region between two materials, as per request from Brian van Straalen.

\section{LLNL update:}

Hank extended VisIt's subset plot to be more efficient for AMR data. Subset plots show where patch boundaries lie. Previously, it was making wireframe and surface subset plots using a general technique that was capable of handling unstructured data. The new implementation creates only a few quads or lines for each patch, which means that performance and 
memory footprint will be much improved. This activity was \#9 on "production quality AMR" from the Spring PMP.

Hank conducted the "VisIt summit", which included participation from VACET personnel (Jeremy Meredith, Sean Ahern, Brad Whitlock, and Gunther Weber) and non-VACET personnel (ten non-VACET developers). The purpose was to consider what the future of the project would look like, given that multiple organizations (including VACET, obviously) are now participating. We decided to continue migration to many of the facilities being provided by the SciDAC Outreach Center's GForge server, including bug tracking and Wikis. Jeremy Meredith will be leading this effort going forward.

Brad has made very good progress in integrating SLIVR with VisIt, although it has not been merged into VisIt's mainline code yet. Through Brad's efforts, SLIVR and a supporting library, TEEM, have been put inside VisIt's build process. SLIVR will be peer to VisIt's other volume rendering techniques (splatting, 3D texturing and ray casting). SLIVR is currently a hardware only mode (meaning that a low resolution data set is rendered using graphics hardware on the VisIt client), but we are also considering a software implementation (meaning that the full resolution is rendered in parallel on the VisIt server). The current implementation works for the one dimensional case, but Brad is currently looking at the case of two dimensional transfer functions. Brad is also looking creating a widget for two dimensional transfer functions, as well as incorporating histograms. Brad has also commented that Marty has been super in supporting this effort. Finally, Brad implemented an interaction method where low resolution data is used while rotating and then a higher resolution form is used when rotation stops.

Tom Treadway has worked on fixing a bug for Macs that was encountered on Phil Colella's laptop. VisIt was unable to start up on this laptop. No additional information as to the cause of the problem is available at the time of the teleconference.

\section{ORNL update:}

Sean has been working on data selections in VisIt. The data selection happens on a per variable basis and this selection is encoded in VisIt's 
pipeline contract. This allows the file format reader to only read in the data that matches the criteria. This was integrated with an effort by the University of Tennessee, Knoxville. This will lay the groundwork for a collaboration with the fast bit query indexing technology coming out of LBNL, if that collaboration (with the SDM center) goes forward.

Jeremy visited LBNL. Gunther set up meeting with both Phil Colella and John Bell's groups. (Gunther also participated in these meetings.) Their conversations focused around two things: (1) debugging AMR codes with VisIt and (2) material interface reconstruction.

For debugging AMR code, Jeremy has completed the infrastructure where Chombo (or any code) can fire up VisIt from a debugger (e.g. gdb). He also wrote a small amount of code that will go into Chombo. During the demonstration that Jeremy gave to Brian, everything worked. Brian said, "this is great, we're moving forward." This completes item \#3 on "production quality AMR" from the Spring PMP. Jeremy and Gunther also sat down with Vince Beckner.

Jeremy also discussed embedded boundaries with Brian, and later with Ken Joy when Jeremy visited LLNL (and Ken was there). It was identified that Chombo will produce "avtMixedVariables" that have two separated values for a single material. Restated, for a given cell, it is possible to separate the per-material values for that cell. Previously, we assumed that for a given cell, the value for a given material would always be the same. With Chombo output, a single material may have multiple values in a given cell (because the material is separated by another material).

Jeremy continued to work on issues for Tech-X. He fixed an ssh tunneling issue that was stopping John Cary from using VisIt. He also made progress on the database options windows, which was a previously identified issue.

Jeremy also met with Andrew Uselton while he was at LBL and they discussed the capabilities being provided by the GForge server. (As previously noted, Jeremy will be leading this effort going forward.)

\section{UCDavis update:}


Christoph has been familiarizing himself with the VisIt code. He and Louis Feng are planning on collaborating on improving VisIt's streamline capability. Christoph has solved a similar problem previously (for fusion customers) and he believes that the accuracy of the solution is extremely important, especially for analysis. The final solution may involve fitting the streamline with curves, instead of using VTK's line segment solution. VisIt is positioned for this.

Sean commented that he would like this functionality to be available in an operator, as well as a plot.

\section{UUtah update:}

Marty is considering putting together a general test application that uses multivariate data. This would require putting together a basic editor. Brad votes for hard coding the transfer function, as he is most interested in how to use the SLIVR library.

Marty wants to continue fixing things in SLIVR. It currently only supports byte data, but he wants to add support for floats in the shader.

We continue to be interested in a distributed memory solution for AMR. We discussed having further discussions at IEEE vis.

Marty wants additional feedback and direction from Dean Williams on the VisTrails front. Brad says that Dean is unavailable these days due to LLNL institutional fire drills. 